\title{
Planning Parking Building Using Flat Slab and Drop Panel as a Replacement Conventional Beam with Analyzing Bending Moment Value \& Sliding Style Based On SNI 1726- 2012
}

\author{
Agyanata Tua Munthe and Guntur Jatmiko \\ Civil Engineering, Mercu Buana University, Jl. Raya Kranggan No.6, \\ Jawa Barat, Indonesia \\ agyanata.umb@gmail.com, gunturjatmiko@gmail.com
}

\begin{abstract}
The construction of a 5-stored parking building is planned to use a flat slab (with drop panels). Flat slab (with drop panel) is a type of two-way plate without beams that directly rests on the column. the flat slab can reduce the height of the structure and construction time. However, flat slabs require plates that are thicker than usual to overcome deflection and punching shears. In this final project, a 5-story reinforced concrete structure will be reviewed with a span of $8 \times 8.3 \mathrm{~m}$. Analysis and design was carried out with the help of the 2016 ETABS program to find the value of intersection between floors, shear moments and forces taking into account the consequences of dead load, super dead load, life, and earthquake (dynamic)
\end{abstract}

Keyword: flat slab, drop panel, parking building, planning

\section{Introduction}

In developing countries like Indonesia, especially in Jakarta with very high mobility, the use of vehicles is very much needed starting from private and public vehicles. At present, the use of private vehicles is still in demand by some people on a more flexible basis because they can walk at any time without having to wait like public transportation. Then the ratio is not balanced between the increasing population of vehicles with available parking space, given that the vacant land in Jakarta is decreasing because it is used for residential and office space, which sometimes has insufficient parking space. So one solution is to build a multi-storey parking building so that it can accommodate a large number of vehicles. In this parking building there are elements consisting of columns, flat slabs with drop panels. All elements are made of reinforced concrete. Reinforced concrete is a concrete reinforced with an area and the number of reinforcement that is not less than the minimum value required with or without prestressing, and is planned based on the assumption that the two materials work together in bearing forces. Flat slabs are thick, evenly thick concrete slabs that transfer loads directly to supporting columns without the help of beams (McCormac, 2000). Drop panel is an increase in the thickness of the plate in the column area that serves to reduce the shear stress caused by the column against the plate. The use of flat slabs with this drop panel system will increase the magnitude of the moment of resistance in places where the negative moments are (More and sawant, 2015). The use of flat slab with this drop panel system will increase the strength of the plate against shear and flexural force and can withstand heavy loads and longer spans.

While the purpose of this research is:

1) Find out how many intersections between floors of the building design

2) Knowing the number of bending moments and shear forces of the building design

\section{Research methods}

Using analysis methods in structural modeling using the 2016 ETABS program to find the value of bending moment and shear force by referring to applicable regulations such as SNI 1726-2012, SNI 1727-2013, SNI 28472013. The flowchart of the study in this study is as follows: 


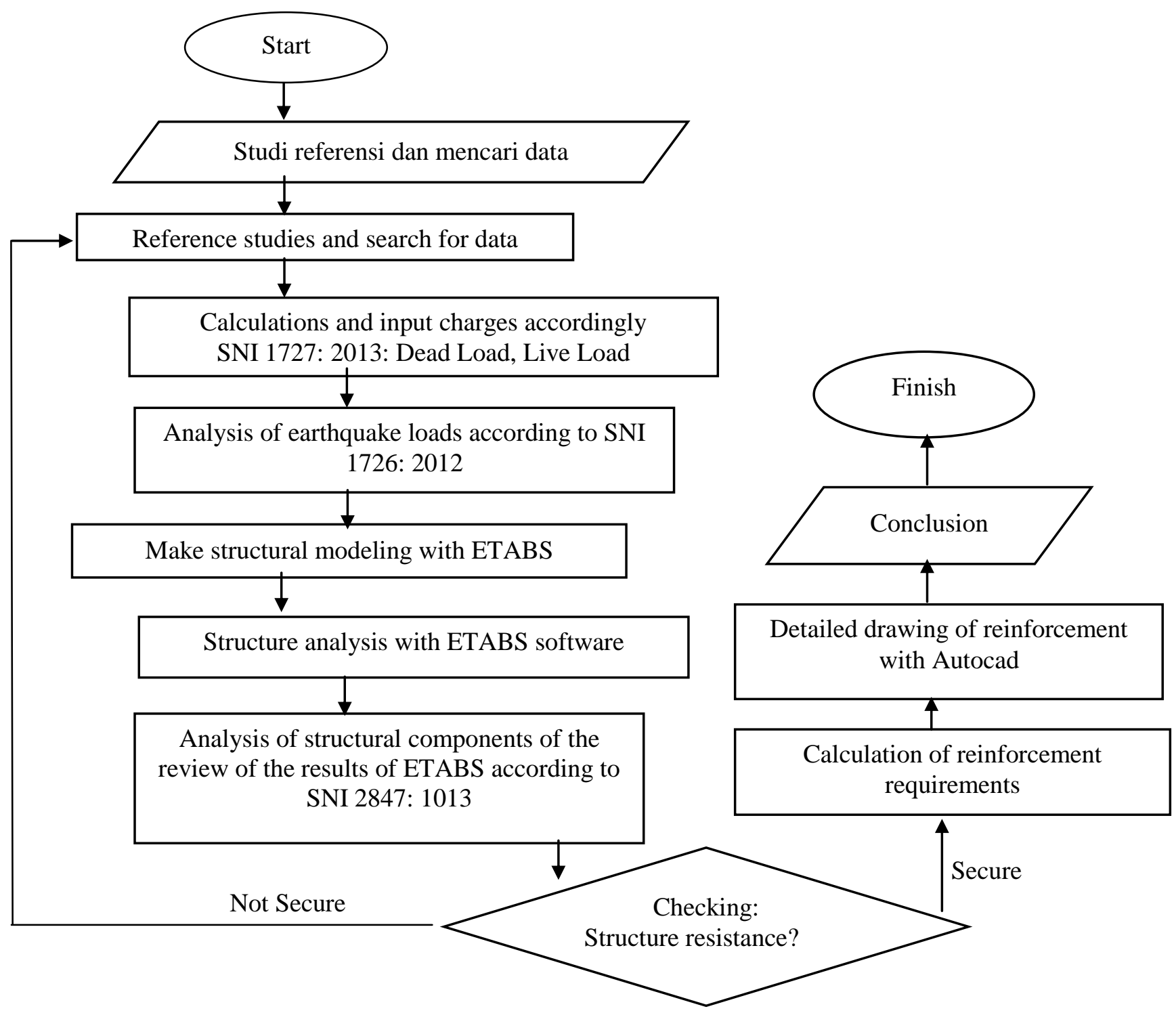

Figure 1. Research Flowchart

\section{Result and Discussion}

\section{Research Analysis Stage}

1. Geometry Structure :

$\checkmark$ Building function : Parking building

$\checkmark$ Number of floors $: 5$ floors (split level)

$\checkmark$ Height per floor $: 3 \mathrm{~m}$

$\checkmark$ Soil type : Medium soil (SD)

2. Material Quality :

$\checkmark$ Reinforced concrete, f'c 35MPa for flat slabs, drop panels, beams and f'c 45MPa for columns

$\checkmark$ Reinforcing steel, Ø8, Ø10, Ø12 mm plain reinforcement BJTP-24 with 240 MPa fy. D10, D13, D16, D19, D22, D25 mm BJTD-40 screw reinforcement with fy $400 \mathrm{MPa}$. And the specific gravity of the iron used is $7850 \mathrm{Kg} / \mathrm{m}^{3}$

3. Existing Dimensions :

$\checkmark$ For Beam B1(300x500), column K1 (1200x1200) dan K2 (900x900)

4. Loading : 
$\checkmark$ Dead load, self weight of its structure (calculated automatically by Etabs)

$\checkmark$ Gravitasi Gravity load is divided into two, namely dead load (instalation ME $=0,25 \mathrm{kN} / \mathrm{m}^{2}$ \& waterproofing with asphalt $=0,28 \mathrm{kN} / \mathrm{m}^{2}$ ) and live load (for parking is set $2 \mathrm{kN} / \mathrm{m}^{2}$ )

$\checkmark$ Gravitasi The gravity load on the slap parapet area is equal to $21,6 \mathrm{kN} / \mathrm{m}^{2}$

5. Premlimenary plates :

$\checkmark$ Flat slab :

- Thickness of directional plates $\mathrm{Y}, \mathrm{L}=9000 \mathrm{~mm}$ (the longest size)

Clean plate length $=>9000-600=8400 \mathrm{~mm}$

For external panels, a minimum thickness of plates $\Rightarrow>8400 / 33=254,54 \mathrm{~mm}$

For panels in a minimum thickness of plates $=>8400 / 36=233,3 \mathrm{~mm}$

The thickness of the plate to be used $260 \mathrm{~mm}$

- Thickness of directional plates X, L=8000mm (the longest size)

Clean plate length $=>8000-600=7400 \mathrm{~mm}$

For external panels, a minimum thickness of plates $=>7400 / 33=224,24 \mathrm{~mm}$

For panels in a minimum thickness of plates $=>7400 / 36=205,5 \mathrm{~mm}$

The thickness of the plate to be used $230 \mathrm{~mm}$

From the 2 directions $\mathrm{Y}$ and $\mathrm{X}$, the largest value is taken $260 \mathrm{~mm}$

- Thickness drop panel $=>260 / 4=65$. Then $260 \mathrm{~mm}+65 \mathrm{~mm}=325 \mathrm{~mm}$

- Determine the extension of the area of the panel drop area with the longest formula L between columns divided by 6 .

6. Earthquake load (E) - elastic earthquake response :
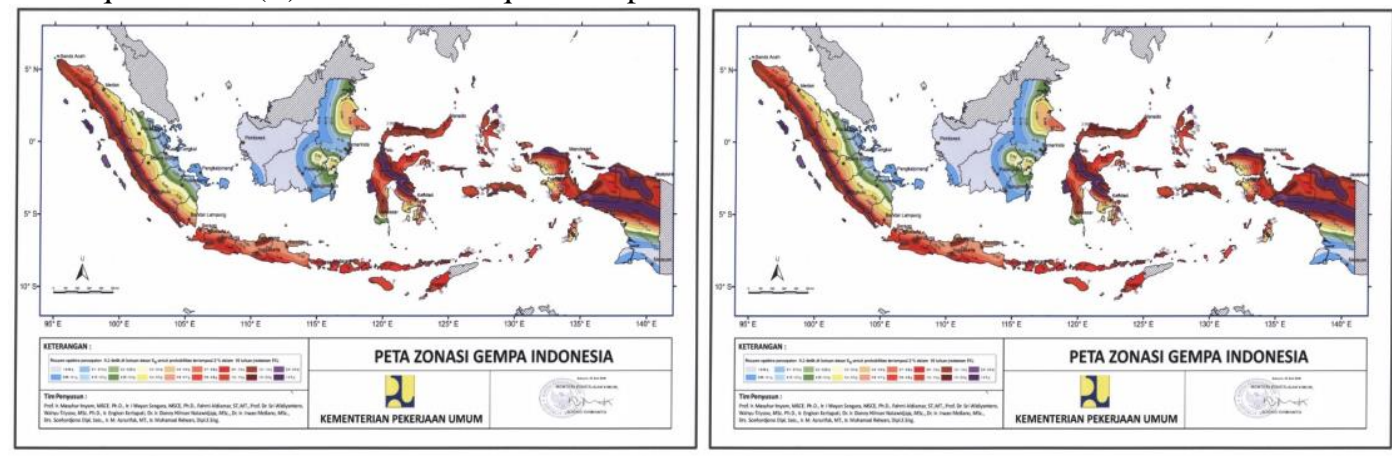

Figure 2. Indonesian Earthquake Map Zone (Ss) \& (Sc)

(Source: Puskim.pu.go.id)

From the Puskim the following data are obtained :

\begin{tabular}{|c|c|c|c|c|c|}
\hline $\mathrm{S}_{\mathrm{s}}$ & 0,669 & $\mathrm{~F}_{\mathrm{a}}$ & 1,265 & $\mathrm{~S}_{\mathrm{DS}}$ & $0,564 \mathrm{~g}$ \\
\hline $\mathrm{S}_{1}$ & 0,295 & $\mathrm{~F}_{\mathrm{y}}$ & 1,811 & $\mathrm{~S}_{\mathrm{D} 1}$ & $0,356 \mathrm{~g}$ \\
\hline
\end{tabular}

The design category is determined by value $S_{D S}$ dan $S_{D 1}$ that is type $D$

$\checkmark \quad$ Vibration period, $\mathrm{T}_{0}=0,126 \mathrm{dt}$ dan $\mathrm{T}_{\mathrm{s}}=0,631 \mathrm{dt}$

7. Design calculation

$\checkmark$ The value of the fundamental period $\Rightarrow T_{a}=0,533$ second \& $T_{\text {maks }}=0,746$ second

$\checkmark$ Periode The period value of Etabs $\quad \Rightarrow \mathrm{T}_{\mathrm{x}}=0,708$ second \& $\mathrm{T}_{\mathrm{y}}=0,659 \operatorname{second}(\mathrm{OK})$

$\checkmark$ Seismic response coefficient values

$\checkmark \quad$ The weight value of the building structure

$\Rightarrow \mathrm{C}_{\mathrm{s}-\mathrm{x}}=0,0785 \& \mathrm{C}_{\mathrm{s}-\mathrm{y}}=0,0844$

$\Rightarrow 70389,82 \mathrm{kN}$

$\checkmark \quad$ Value of basic seismic shear forces

$\checkmark$ Exponent value (k)

$\checkmark \quad$ Lateral earthquake force values

$\checkmark \quad$ Selection of types

$\Rightarrow \mathrm{V}_{\mathrm{s}-\mathrm{x}}=5525,6 \mathrm{kN} \& \mathrm{~V}_{\mathrm{s}-\mathrm{y}}=5940,9 \mathrm{kN}$

$\Rightarrow \mathrm{T}_{\mathrm{x}}=1,104 \& \mathrm{~T}_{\mathrm{y}}=1,07$

$\Rightarrow \mathrm{F}_{\mathrm{x}}=5525,6 \mathrm{kN} \& \mathrm{~F}_{\mathrm{y}}=5940,9 \mathrm{kN}$

$\checkmark$ Basic shear force scale

$\Rightarrow 4,9 \%$ entered into CQC

$\checkmark$ Intersection between floors due to earthquake loads $\mathrm{X}$ and $\mathrm{Y}$ 
VOLUME 2 | NUMBER 3 | MAY 2020 Available online at http://proceedings.worldconference.id. ISSN: 2656-1174 (online)

\begin{tabular}{|l|c|c|c|c|c|c|}
\hline \multirow{2}{*}{ Floor } & $\mathrm{h}$ & $\mathrm{X}$-Dir & Y-Dir & $\Delta_{\text {izin }}=0,015 \mathrm{~h}$ & \multicolumn{2}{c|}{ Check } \\
\cline { 2 - 7 } & $(\mathrm{mm})$ & $(\mathrm{mm})$ & $(\mathrm{mm})$ & $(\mathrm{mm})$ & X-Dir & Y-Dir \\
\hline P5A & 15000 & 0,256 & 0,027 & 22,5 & OK & OK \\
\hline P5 & 13500 & 0,223 & 0,009 & 22,5 & OK & OK \\
\hline P4A & 12000 & 0,19 & 0,02 & 22,5 & OK & OK \\
\hline P4 & 10500 & 0,157 & 0,007 & 22,5 & OK & OK \\
\hline P3A & 9000 & 0,124 & 0,014 & 22,5 & OK & OK \\
\hline P3 & 7500 & 0,093 & 0,004 & 22,5 & OK & OK \\
\hline P2A & 6000 & 0,064 & 0,007 & 22,5 & OK & OK \\
\hline P2 & 4500 & 0,039 & 0,002 & 22,5 & OK & OK \\
\hline P1A & 3000 & 0,019 & 0,002 & 22,5 & OK & OK \\
\hline P1 & 1500 & 0,005 & 0,000248 & 22,5 & OK & OK \\
\hline Base & 0 & 0 & 0 & 0 & & \\
\hline
\end{tabular}

$\checkmark \quad$ Output shear and moment values

\begin{tabular}{|c|c|c|}
\hline Lantai & V maks & V min \\
\hline Base & & \\
\hline P1 & 472,05 & $-1109,658$ \\
\hline P1A & 476,713 & $-1043,016$ \\
\hline P2 & 835,537 & $-1261,551$ \\
\hline P2A & 858,514 & $-1316,273$ \\
\hline P3 & 789,126 & $-1324,012$ \\
\hline P3A & 887,051 & $-1384,624$ \\
\hline P4 & 802,956 & $-1327,791$ \\
\hline P4A & 871,651 & $-1358,133$ \\
\hline P5 & 764,171 & $-1332,95$ \\
\hline P5A & 853,337 & $-1276,898$ \\
\hline
\end{tabular}

\begin{tabular}{|l|c|c|c|c|}
\hline \multirow{2}{*}{ Lantai } & \multicolumn{2}{|c|}{ Arah X } & \multicolumn{2}{c|}{ Arah Y } \\
\cline { 2 - 5 } & M max & M min & M max & M min \\
\hline Base & & & & \\
\hline P1 & 80,307 & $-489,473$ & 79,73 & $-580,26$ \\
\hline P1A & 235,517 & $-530,764$ & 247,289 & $-630,154$ \\
\hline P2 & 190,708 & $-551,333$ & 256,59 & $-659,61$ \\
\hline P2A & 319,738 & $-578,021$ & 282,405 & $-686,357$ \\
\hline P3 & 236,877 & $-575,467$ & 326,877 & $-692,452$ \\
\hline P3A & 328,285 & $-588,671$ & 328,179 & $-702,975$ \\
\hline P4 & 50,336 & $-578,859$ & 328,47 & $-694,669$ \\
\hline P4A & 324,552 & $-585,06$ & 323,885 & $-695,009$ \\
\hline P5 & 49,887 & $-564,647$ & 324,31 & $-697,134$ \\
\hline P5A & 261,125 & $-567,894$ & 283,987 & $-693,891$ \\
\hline
\end{tabular}

$\checkmark \quad$ The reviewed flat slab area and the moment of etabs

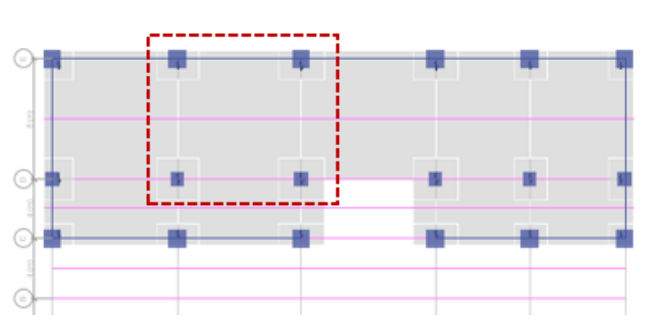

\begin{tabular}{|c|c|c|c|}
\hline \multicolumn{4}{|c|}{ Arah X (memendek) } \\
\hline \multicolumn{2}{|c|}{ Lajur kolom } & \multicolumn{2}{c|}{ Lajur Tengah } \\
\hline Tumpuan & Lapangan & Tumpuan & Lapangan \\
\hline$-312,855$ & $-15,657$ & 13,235 & 12,521 \\
\hline \multicolumn{4}{|c|}{ Arah Y (memanjang) } \\
\hline \multicolumn{3}{|c|}{ Lajur kolom } & Lajur Tengah \\
\hline Tumpuan & Lapangan & Tumpuan & Lapangan \\
\hline$-319,506$ & $-3,536$ & 16,546 & 28,3 \\
\hline
\end{tabular}

Reinforcement calculation steps

- 1-way slide check for critical cross sections A-A (X)

$\mathrm{Vu} \quad=312,855 \mathrm{kN}=31,9$ ton

$\emptyset V c \quad=0,75 \times \sqrt{\frac{f^{\prime} \varepsilon}{6}} \times \mathrm{b} \times \mathrm{d}$

$\emptyset V c \quad=0,75 \times \sqrt{\frac{35}{6}} \times 8 \times 0,233=137,84$ ton

$V u<\varnothing V c(O K)$ 
- $\quad$ 1-way slide check for critical cross sections B-B (Y)

$\mathrm{Vu} \quad=319,506 \mathrm{kN}=32,58$ ton

$\emptyset V c \quad=0,75 \times \sqrt{\frac{f^{\prime} c}{6}} \times \mathrm{b} \times \mathrm{d}$

$\emptyset V c \quad=0,75 \times \sqrt{\frac{35}{6}} \times 8,3 \times 0,233=143,01$ ton

$V u<\varnothing V c(O K)$

- Check 2-way slide

$\mathrm{Vu} \quad=319,506 \mathrm{kN}=32,58$ ton

Bc $\quad=900 / 900=1$

as $\quad=40$

$\mathrm{b}_{\mathrm{o}} \quad=2 \mathrm{x}(1016,75+1016,75)=4067 \mathrm{~mm}=4,067 \mathrm{~m}$

$V_{c(a)}=\frac{1}{6} \times\left(1+\frac{2}{B c}\right) \times \sqrt{f^{\prime} c} \times b_{o} \times d$

$=\frac{1}{6} \times\left(1+\frac{2}{1}\right) \times \sqrt{35} \times 4,067 \times 0,233=2,8=280$ ton

$V_{c(b)}=\left(\frac{a s x d}{12 \mathrm{bo}}+\frac{1}{6}\right) \times \sqrt{f^{\prime} c} \times \mathrm{b}_{\mathrm{o}} \times \mathrm{d}$

$=\left(\frac{40 \times 0,233}{12 \times 4,067}+\frac{1}{6}\right) \times \sqrt{35} \times 4,067 \times 0,233=2,0049=200$ ton

$V_{c(c)}=\frac{1}{3} \times \sqrt{f^{\prime} c} \times b_{0} \times d=\frac{1}{3} \times \sqrt{35} \times 4,067 \times 0,233=1,86=186$ ton

Take the smallest $\mathrm{Vc}$ value, such that

$\emptyset V c \quad=0,75 \times 186=139,5$ ton

Then, $V u \quad=32,58$ ton $<\varnothing V c=139,5$ ton, the plate shear capacity is sufficient to carry two-way shear

- Calculate the effective height of a floor plate (d)

Concrete blanket for floor plates $20 \mathrm{~mm}\left(\mathrm{t}_{\mathrm{s}}\right)$

Principal reinforcement used D13mm

$\mathrm{d} \quad=\mathrm{h}-\mathrm{t}_{\mathrm{s}}-0,5 \times \mathrm{D}$

$=260-20-0,5 \times 13=233,5 \mathrm{~mm}$ (long direction)

$\mathrm{d}=\mathrm{h}-\mathrm{t}_{\mathrm{s}}-\mathrm{D}-0,5 \times \mathrm{D}$

$=260-20-13-0,5 \times 13=220,5 \mathrm{~mm}$ (short direction)

- Calculate the value $\boldsymbol{\beta}_{\boldsymbol{I}}$

For f'c $\leq 30 \mathrm{MPa}, \beta_{1}=0,85$

For $\mathrm{f}^{\prime} \mathrm{c} 30<\mathrm{f}^{\prime} \mathrm{c}<55 \mathrm{MPa}, \beta_{1}=0,85-0,008\left(\mathrm{f}^{\prime} \mathrm{c}-30\right)$

For $\mathrm{f}^{\prime} \mathrm{c}>55 \mathrm{Mpa}, \beta_{1}=0,65$

Then,

f'c $35 \mathrm{MPa}, \beta_{1}=0,85-0,008(35-30)=0,81$

$\rho_{b}=\frac{0.85 \times \beta x f^{c} c}{f y}\left(\frac{600}{600+f y}\right)=\frac{0,85 \times 0,81 \times 35}{400}\left(\frac{600}{600+400}\right)=0,0361$

- $\quad$ Calculate the amount of the minimum and maximum reinforcement ratio

$\rho_{g}=0,0018$ (bersumber SNI 2847:2013 pasal 7.12.2.1)

$\rho_{\text {maks }}=0,75 \times p_{b}=0,75 \times 0,0361=0,027$

- Calculating the reinforcement of the column shortening direction (pedestal)

Calculation of the principal reinforcement in the column lane area uses reinforcement D $13 \mathrm{~mm}$.

$\mathrm{M}_{\mathrm{o}}=312,855 \mathrm{kNm}$

$M_{n}=\left(\frac{M_{0}}{\varphi}\right)=\left(\frac{312,855}{0.9}\right)=347,62 \mathrm{kNm}$

$R_{n}=\left(\frac{M_{n}}{b x d^{2}}\right)=\left(\frac{347610000}{1000 \times 220,5^{2}}\right)=7,15$

$m=\left(\frac{\text { fy }}{0,85 \times f^{\prime} c}\right)=\left(\frac{400}{0,85 \times 35}\right)=13,45$

Reinforcement ratio 
$\rho=\frac{1}{m}\left[1-\sqrt{1-\frac{2(m)\left(R_{n}\right)}{f y}}\right]$

$\rho=\frac{1}{13_{\imath} 45}\left[1-\sqrt{1-\frac{2(13,45)(7,15)}{400}}\right]=0,0208$

Area reinforcement needed

$$
\begin{aligned}
& \text { As } \min =\rho_{g} \times \mathrm{b} \times \mathrm{t} \quad=0,0018 \times 1000 \times 260=468 \mathrm{~mm}^{2} \\
& \text { As } \quad=\rho \times \mathrm{b} \mathrm{x} \mathrm{d}^{2} \quad=0,0208 \times 1000 \times 220,5=4581,067 \mathrm{~mm}^{2}
\end{aligned}
$$

Then used As $=4581,067 \mathrm{~mm}^{2}$

Distance between reinforcement

$S=\left(\frac{0.25 \times \pi x \varphi^{2} x b}{A_{\Omega}}\right)=\left(\frac{0,25 \times 3,14 \times 13^{2} x 1000}{4581,067}\right)=28,95 \mathrm{~mm} \sim 30 \mathbf{~ m m}$

$S=\left(\frac{0.25 \times \pi x \varphi^{2} x b}{s}\right)=\left(\frac{0.25 \times 3.14 \times 13^{2} \times 1000}{30}\right)=4422,16>\mathbf{3 0} \mathbf{~ m m}(\mathbf{O K})$

Then the reinforcement of column lane shortening (Footing) is used= D13 - $30 \mathbf{~ m m}$

- Calculate the reinforcement of the column shortening direction (field)

Calculation of the principal reinforcement in the column lane area uses reinforcement D $13 \mathrm{~mm}$.

$\mathrm{M}_{\mathrm{o}}=15,657 \mathrm{kNm}$

$M_{n}=\left(\frac{M_{0}}{\varphi}\right)=\left(\frac{15,657}{0.9}\right)=17,4 \mathrm{kNm}$

$R_{n}=\left(\frac{M_{n}}{b x d^{2}}\right)=\left(\frac{17400000}{1000 \times 220,5^{2}}\right)=0,36$

$m=\left(\frac{\text { fy }}{0,85 \times f^{\prime} c}\right)=\left(\frac{400}{0,85 \times 35}\right)=13,45$

Reinforcement ratio

$\rho=\frac{1}{m}\left[1-\sqrt{1-\frac{2(m)\left(R_{n}\right)}{f y}}\right]$

$\rho=\frac{1}{13,45}\left[1-\sqrt{1-\frac{2(13,45)(0,36)}{400}}\right]=0,0009$

Area reinforcement needed
As min $=\rho_{g} \times \mathrm{b} \times \mathrm{t}$
$=0,0018 \times 1000 \times 260=468 \mathrm{~mm}^{2}$
As $=\rho \times \mathrm{bxd} \quad=0,0009 \times 1000 \times 220,5=198,442 \mathrm{~mm}^{2}$

Because As $\min >$ As, then used As $\min =468 \mathrm{~mm}^{2}$

Distance between reinforcement

$$
\begin{aligned}
& S=\left(\frac{0.25 \times \pi x \varphi^{2} x b}{A_{S}}\right)=\left(\frac{0,25 \times 3,14 \times 13^{2} \times 1000}{468}\right)=283,47 \mathrm{~mm} \sim 290 \mathbf{~ m m} \\
& S=\left(\frac{0.25 \times \pi x \varphi^{2} x b}{S}\right)=\left(\frac{0.25 \times 3.14 \times 13^{2} \times 1000}{290}\right)=457,46>290 \mathbf{~ m m}(\text { OK })
\end{aligned}
$$

Then used the direction of the column shortening direction column (Field) $=\mathbf{D 1 3} \mathbf{- 2 9 0} \mathbf{~ m m}$

- Calculate the reinforcement of the shortened center lane (pedestal)

Calculation of the principal reinforcement in the column lane area uses reinforcement D $13 \mathrm{~mm}$. $\mathrm{M}_{\mathrm{o}}=13,235 \mathrm{kNm}$

$$
\begin{aligned}
& M_{n}=\left(\frac{M_{0}}{\varphi}\right)=\left(\frac{13,235}{0,9}\right)=14,71 \mathrm{kNm} \\
& R_{n}=\left(\frac{M_{n}}{b x d^{2}}\right)=\left(\frac{14710000}{1000 \times 220,5^{2}}\right)=0,30 \\
& m=\left(\frac{f y}{0,85 x f^{\circ} c}\right)=\left(\frac{400}{0,85 \times 35}\right)=13,45
\end{aligned}
$$

Reinforcement ratio 
$\rho=\frac{1}{m}\left[1-\sqrt{1-\frac{2(m)\left(R_{n}\right)}{f y}}\right]$

$\rho=\frac{1}{13,45}\left[1-\sqrt{1-\frac{2(13,45)(0,3)}{400}}\right]=0,0008$

Area reinforcement needed

$$
\begin{array}{llll}
\text { As } \min & =\rho_{g} \times \mathrm{b} \mathrm{x} \mathrm{t} & =0,0018 \times 1000 \times 260 & =468 \mathrm{~mm}^{2} \\
\text { As } & =\rho \times \mathrm{b} \times \mathrm{d} & =0,0008 \times 1000 \times 220,5 & =167,586 \mathrm{~mm}^{2}
\end{array}
$$

Because As min > As, then used As $\min =468 \mathrm{~mm}^{2}$

Distance between reinforcement

$S=\left(\frac{0.25 \times \pi x \varphi^{2} x b}{A_{\Phi}}\right)=\left(\frac{0,25 \times 3,14 \times 13^{2} x 1000}{468}\right)=283,47 \mathrm{~mm} \sim 290 \mathbf{~ m m}$

$S=\left(\frac{0.25 \times \pi x \varphi^{2} x b}{s}\right)=\left(\frac{0.25 \times 3.14 \times 13^{2} \times 1000}{290}\right)=457,46>290 \mathbf{~ m m}($ OK)

Maka digunakan tulangan arah memendek lajur tengah $($ Tumpuan $)=$ D13 -290 mm

- Calculate the shortened center lane reinforcement (field)

Calculation of the main reinforcement in the column path are D $13 \mathrm{~mm}$.

$\mathrm{M}_{\mathrm{o}}=12,521 \mathrm{kNm}$

$M_{n}=\left(\frac{M_{0}}{\varphi}\right)=\left(\frac{12,521}{0.9}\right)=13,91 \mathrm{kNm}$

$R_{n}=\left(\frac{M_{n}}{b x d^{2}}\right)=\left(\frac{14710000}{1000 \times 220,5^{2}}\right)=0,29$

$m=\left(\frac{f y}{0,85 \times f^{\prime c}}\right)=\left(\frac{400}{0,85 \times 35}\right)=13,45$

Reinforcement ratio

$\rho=\frac{1}{m}\left[1-\sqrt{1-\frac{2(m)\left(R_{n}\right)}{f y}}\right]$

$\rho=\frac{1}{13,45}\left[1-\sqrt{1-\frac{2(13,45)(0,29)}{400}}\right]=0,0007$

Area reinforcement needed
As $\min =\rho_{g} \times \mathrm{b} \times \mathrm{t}$
$=0,0018 \times 1000 \times 260=468 \mathrm{~mm}^{2}$
As $\quad=\rho \times \mathrm{b} \times \mathrm{d} \quad=0,0007 \times 1000 \times 220,5=158,501 \mathrm{~mm}^{2}$

Becuase As min $>$ As, then used As $\min =468 \mathrm{~mm}^{2}$

Distance between reinforcement

$$
\begin{aligned}
& S=\left(\frac{0.25 \times \pi x \varphi^{2} x b}{A_{S}}\right)=\left(\frac{0,25 \times 3,14 \times 13^{2} \times 1000}{468}\right)=283,47 \mathrm{~mm} \sim 290 \mathbf{~ m m} \\
& S=\left(\frac{0.25 \times \pi x \varphi^{2} x b}{S}\right)=\left(\frac{0.25 \times 3.14 \times 13^{2} \times 1000}{290}\right)=457,46>290 \mathbf{~ m m}(\text { OK) }
\end{aligned}
$$

Then used the direction bar shortening the middle lane (Field) $=$ D13 - $290 \mathbf{~ m m}$

$\checkmark \quad$ Recap the flat slab reinforcement is shortened (X)

\begin{tabular}{|l|c|c|}
\hline Momen yang di tinjau & Diameter & Jarak \\
\hline Tumpuan (lajur kolom) & D 13 & 30 \\
\hline Lapangan (lajur kolom) & D 13 & 290 \\
\hline Tumpuan (lajur tengah) & D 13 & 290 \\
\hline Lapangan (lajur tengah) & D 13 & 290 \\
\hline
\end{tabular}

$\checkmark$ Using the Mo value according to the output etabs and the same calculation steps with the reinforcement of the flat slab in the shortening direction, it is obtained the Recap of the flat slab reinforcement in the longitudinal direction $(\mathrm{Y})$ 


\section{4 \\ VOLUME 2 | NUMBER 3 | MAY 2020}

Available online at http://proceedings.worldconference.id.

ISSN: 2656-1174 (online)

\begin{tabular}{|l|c|c|}
\hline Momen yang di tinjau & Diameter & Jarak \\
\hline Tumpuan (lajur kolom) & D 13 & 30 \\
\hline Lapangan (lajur kolom) & D 13 & 290 \\
\hline Tumpuan (lajur tengah) & D 13 & 290 \\
\hline Lapangan (lajur tengah) & D 13 & 290 \\
\hline
\end{tabular}

Flat slab reinforcement image shortening $(\mathrm{X})$ and longitudinal direction (Y)

\begin{tabular}{|c|c|c|c|}
\hline \multicolumn{4}{|c|}{ Arah X (memendek) } \\
\hline \multicolumn{3}{|c|}{ Lajur kolom } & \multicolumn{2}{c|}{ Lajur Tengah } \\
\hline Tumpuan & Lapangan & Tumpuan & Lapangan \\
\hline 232,261 & 55,93 & 79,84 & 5,12 \\
\hline \multicolumn{4}{|c|}{ Arah Y (memanjang) } \\
\hline \multicolumn{3}{|c|}{ Lajur kolom } & Lajur Tengah \\
\hline Tumpuan & Lapangan & Tumpuan & Lapangan \\
\hline 182,826 & 68,12 & 78,85 & 9,29 \\
\hline
\end{tabular}
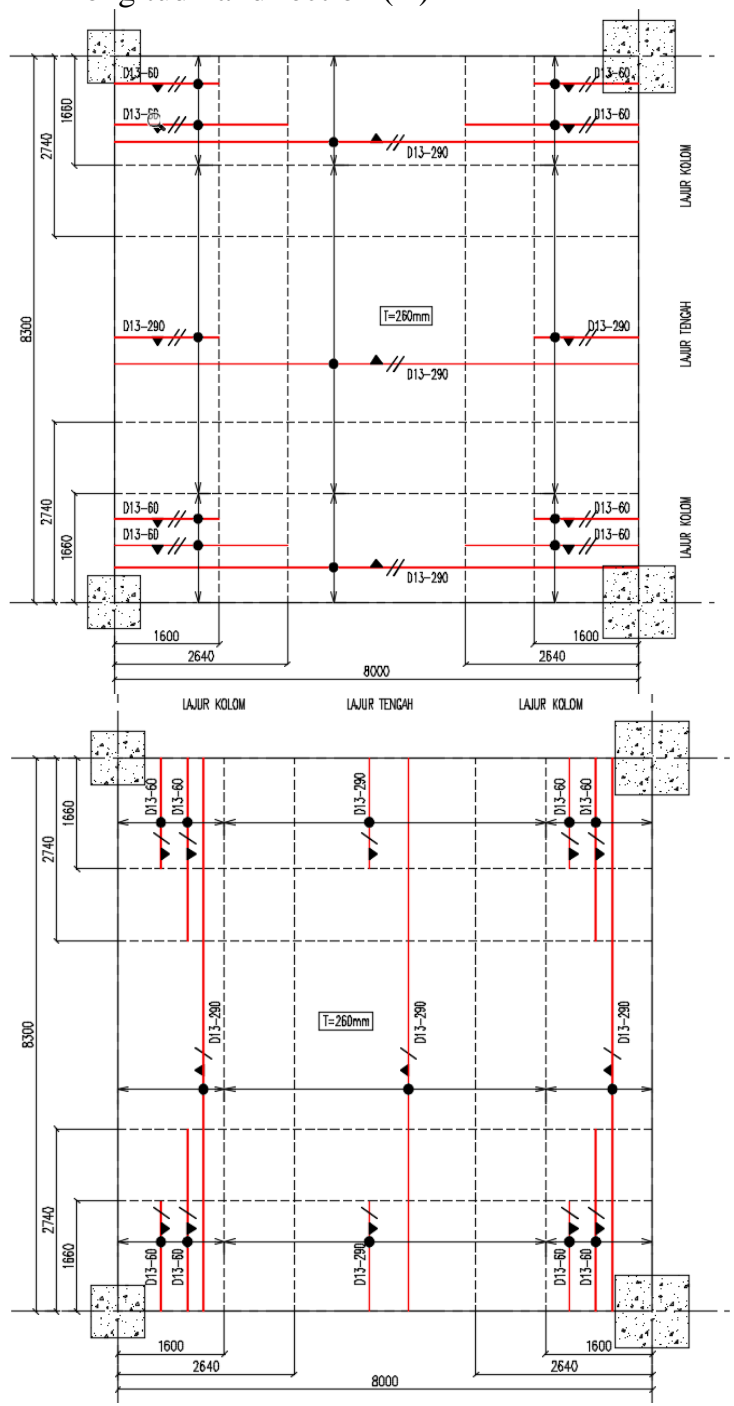

$\checkmark \quad$ Drop The reviewed panel drop area and the moment output from etabs

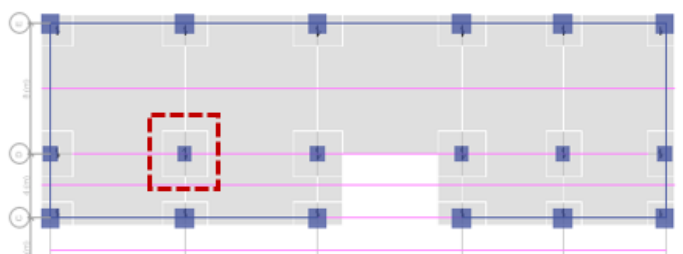


- 1 -way slide check for critical cross sections A-A (X)

$\mathrm{Vu} \quad=232,261 \mathrm{kN}=23,684$ ton

$\emptyset V c \quad=0,75 \times \sqrt{\frac{f^{\prime} c}{6}} \times \mathrm{b} \times \mathrm{d}$

$\varnothing V c \quad=0,75 \times \sqrt{\frac{35}{6}} \times 2,8 \times 0,289=59,84$ ton

$V u<\varnothing V c(O K)$

- 1-way slide check for critical cross sections B-B (Y)

$\mathrm{Vu} \quad=182,826 \mathrm{kN}=18,64$ ton

$\emptyset V c \quad=0,75 \times \sqrt{\frac{f^{r} \varepsilon}{6}} \times \mathrm{b} \times \mathrm{d}$

$\varnothing V c \quad=0,75 \times \sqrt{\frac{35}{6}} \times 2,8 \times 0,289=59,84$ ton

$V u<\varnothing V c(O K)$

- Check 2-way slide

$\mathrm{Vu} \quad=232,261 \mathrm{kN}=23,684$ ton

Bc $\quad=900 / 900=1$

as $\quad=40$

$\mathrm{b}_{\mathrm{o}} \quad=2 \mathrm{x}(1044,5+1044,5)=4,178 \mathrm{~mm}=4,2 \mathrm{~mm}$

$V_{c(a)}=\frac{1}{6} \times\left(1+\frac{2}{B c}\right) \times \sqrt{f^{\prime} c} \times b_{o} \times d$

$=\frac{1}{6} \times\left(1+\frac{2}{1}\right) \times \sqrt{35} \times 4,2 \times 0,289=3,590=359$ ton

$V_{c(b)}=\left(\frac{a s x d}{12 b_{o}}+\frac{1}{6}\right) \times \sqrt{f^{\prime} c} \times b_{o} \times d$

$=\left(\frac{40 \times 0,289}{12 \times 4,2}+\frac{1}{6}\right) \times \sqrt{35} \times 4,2 \times 0,289=2,84=284,34$ ton

$V_{c(c)} \quad=\frac{1}{3} \times \sqrt{f^{\prime} c} \times b_{0} \times d=\frac{1}{3} \times \sqrt{35} \times 4,2 \times 0,289=2,39=239,4$ ton

Take the smallest $\mathrm{Vc}$ value, such that

$\varnothing V c \quad=0,75 \times 239,4=179,55$ ton

Then, $V u \quad=23,684$ ton $<\varnothing V c=179,55$ ton, the plate shear capacity is sufficient to carry two-way shear

- Calculate the effective height of the floor plate (d)Selimut beton untuk pelat lantai $20 \mathrm{~mm}\left(\mathrm{t}_{\mathrm{s}}\right)$. Main reinforcement used D16mm

$$
\begin{aligned}
\mathrm{d} & =\mathrm{h}-\mathrm{t}_{\mathrm{s}}-\mathrm{D} \\
\mathrm{d} & =325-20-16=289 \mathrm{~mm} \text { (lajur kolom) } \\
& =\mathrm{h}-\mathrm{t}_{\mathrm{s}}-\mathrm{D} \\
& =260-20-16=224 \mathrm{~mm} \text { (lajur tengah) }
\end{aligned}
$$

- Calculate the value $\boldsymbol{\beta}_{1}$

For $\mathrm{f}^{\prime} \mathrm{c} \leq 30 \mathrm{MPa}, \beta_{1}=0,85$

For $\mathrm{f}^{\prime} \mathrm{c} 30<\mathrm{f}^{\prime} \mathrm{c}<55 \mathrm{MPa}, \beta_{1}=0,85-0,008\left(\mathrm{f}^{\prime} \mathrm{c}-30\right)$

For f'c $>55 \mathrm{Mpa}, \beta_{1}=0,65$

Then,

f'c $35 \mathrm{MPa}, \beta_{1}=0,85-0,008(35-30)=0,81$

$\rho_{b}=\frac{0.85 x \beta x f^{\prime c} c}{f y}\left(\frac{600}{600+f y}\right)=\frac{0,85 \times 0,81 \times 35}{400}\left(\frac{600}{600+400}\right)=0,0361$

- Calculate the amount of the minimum and maximum reinforcement ratio $\rho_{g}=0,0018$ (based on SNI 2847:2013 pasal 7.12.2.1)

$\rho_{\text {maks }}=0,75 \times p_{b}=0,75 \times 0,0361=0,027$

- Calculating the reinforcement of the column shortening direction (pedestal)

Calculation of the principal reinforcement in the column lane area uses reinforcement D $16 \mathrm{~mm}$.

$\mathrm{M}_{\mathrm{o}}=232,261 \mathrm{kNm}$ 
$M_{n}=\left(\frac{M_{0}}{\varphi}\right)=\left(\frac{232,261}{0.9}\right)=258,067 \mathrm{kNm}$

$R_{n}=\left(\frac{M_{n}}{b x d^{2}}\right)=\left(\frac{258067000}{1000 \times 289^{2}}\right)=3,09$

$m=\left(\frac{\text { fy }}{0,85 \times f^{\prime c}}\right)=\left(\frac{400}{0,85 \times 35}\right)=13,45$

Reinforcement ratio

$\rho=\frac{1}{m}\left[1-\sqrt{1-\frac{2(m)\left(R_{n}\right)}{f y}}\right]$

$\rho=\frac{1}{13,45}\left[1-\sqrt{1-\frac{2(13,45)(3,09)}{400}}\right]=0,0082$

Area reinforcement needed
As $\min =\rho_{g} \times \mathrm{b} \times \mathrm{t}$
$=0,0018 \times 1000 \times 325$
$=585 \mathrm{~mm}^{2}$
As $\quad=\rho \times \mathrm{x} \mathrm{x} \mathrm{d}^{2}$
$=0,0082 \times 1000 \times 289$
$=2369,8 \mathrm{~mm}^{2}$

Then used As $=2369,8 \mathrm{~mm}^{2}$

Distance between reinforcement

$S=\left(\frac{0.25 \times \pi x \varphi^{2} x b}{A \varsigma}\right)=\left(\frac{0,25 \times 3,14 \times 16^{2} x 1000}{2369,8}\right)=84,8 \mathrm{~mm} \sim 90 \mathrm{~mm}$

$A s=\left(\frac{0.25 \times \pi x \varphi^{2} x b}{s}\right)=\left(\frac{0.25 \times 3.14 \times 16^{2} \times 1000}{90}\right)=2232,8>90 \mathbf{~ m m}(\mathbf{O K})$

Then the reinforcement of column lane shortening (Footing) is used = D16 - $90 \mathrm{~mm}$

Then use the same steps as the calculation in the slab

$\checkmark$ Recap the shortening direction drop bar reinforcement (X)

\begin{tabular}{|c|c|c|}
\hline Momen yang di tinjau & Diameter & Jarak \\
\hline Tumpuan (lajur kolom) & D 16 & 90 \\
\hline Lapangan (lajur kolom) & D 16 & 350 \\
\hline Tumpuan (lajur tengah) & D 16 & 250 \\
\hline Lapangan (lajur tengah) & D 16 & 350 \\
\hline
\end{tabular}

$\checkmark$ Using the Mo value according to the output etabs and the same calculation steps as the reinforcement of the shortening direction panel then the longitudinal direction of the drop panel reinforcement is obtained (Y)

\begin{tabular}{|l|c|c|}
\hline Momen yang di tinjau & Diameter & Jarak \\
\hline Tumpuan (lajur kolom) & D 16 & 110 \\
\hline Lapangan (lajur kolom) & D 16 & 300 \\
\hline Tumpuan (lajur tengah) & D 16 & 260 \\
\hline Lapangan (lajur tengah) & D 16 & 350 \\
\hline
\end{tabular}

$\checkmark \quad$ Image of reinforcement drop panel shortening (X) and longitudinal direction (Y) 

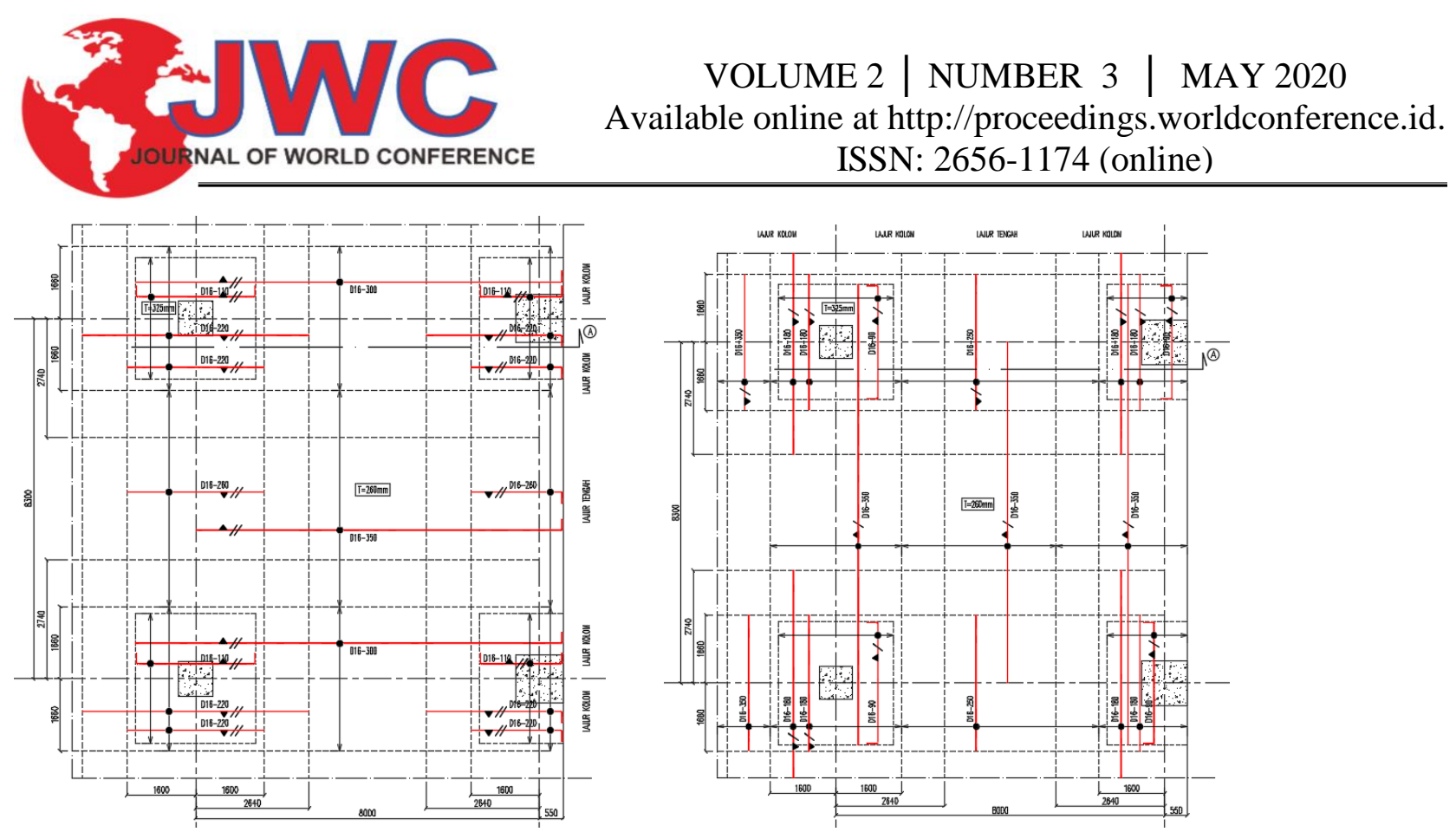

\section{Conclussion}

\section{a. Conclusion}

From the results and analysis of flat slab and drop panel calculations can be concluded:

1. This parking building with flat slab and Drop panel 5 floors is feasible to use because the intersection between floors is $0.10 \mathrm{~mm}$ for $\mathrm{X}$ direction and $0.008 \mathrm{~mm}$ for $\mathrm{Y}$ direction taken on average from 5 floors. This value is below the permit value limit of $22.5 \mathrm{~cm}$.

2. Thick flat slab 260mm and Drop panel 325mm using reinforcement D13-30, D13-290, D16-90, D16-110, D16250, D16-260, D16-300 and D16-350

3. The smallest panel drop size from $1650 \times 1950 \mathrm{~mm}$ to $2800 \times 3000 \mathrm{~mm}$ (floor plan attached)

4. For the biggest moment value on the P3A floor with:

$\begin{array}{lll}\checkmark & \text { M max direction X } & =32,285 \\ \checkmark & \text { M min direction X } & =-588,671 \\ \checkmark & \text { M max direction Y } & =328,179 \\ \checkmark & \text { M min direction Y } & =-702,975\end{array}$

5. For flat slabs, slide one-way cross section critical $\mathrm{A}-\mathrm{A}(\mathrm{X}), \mathrm{Vu}\langle\emptyset \mathrm{Vc}(\mathrm{OK})=>31.9$ tons $<137.84$ tons. For one-way sliding critical cross sections $\mathrm{B}-\mathrm{B}(\mathrm{Y}), \mathrm{Vu}<\emptyset \mathrm{Vc}(\mathrm{OK}) \Rightarrow 32.58$ tons $<143.01$ tons. For the two-way cross section critical A-A $(\mathrm{X}), \mathrm{Vu}\langle\emptyset \mathrm{Vc}(\mathrm{OK})=>32.58$ tons $<139.5$ tons

6. For drop panels, slide one-way critical cross sections $\mathrm{A}-\mathrm{A}(\mathrm{X}), \mathrm{Vu}\langle\emptyset \mathrm{Vc}(\mathrm{OK})=>23,684$ tons $<59.84$ tons. For one-way sliding critical cross section $\mathrm{B}-\mathrm{B}(\mathrm{Y}), \mathrm{Vu}\langle\emptyset \mathrm{Vc}(\mathrm{OK}) \Rightarrow>18.64$ tons $<59.84$ tons. For the two-way sliding cross section critical A-A $(\mathrm{X}), \mathrm{Vu}<\varnothing \mathrm{Vc}(\mathrm{OK})=>23,684$ tons $<179.55$ tons

\section{b. Suggestion}

For further research development regarding flat slabs and drop panels, it is recommended to:

1. In this Final Project do not try various assumptions about the thickness of the flat slab and drop panel due to time constraints. Maybe this can be a material for further researchers to conduct more detailed research in order to obtain an efficient thickness

2. Taking into account the use of shear reinforcement, such as shear stud or structural shearhead on flat plates and flat slabs (drop panels).

3. Calculating the reinforcement ratio

\section{References}

[1] Asy-Syifa, Aulia Rahman. Tarigan, Johannes (2017): Flat slab analysis using square column drop panels with variations in life load loading, Universitas Sumatera Utara, Medan

[2] Budiman, Akhmad Arif (2018): Final task of redesigning the MCC building structure (main control center) using the flat slab system of the Jakarta Corridor 1 LRT project (phase 1) ivory-velodrome coconut, Mercu Buana University, Jakarta 
[3] Burhanuddin, Dody. Wahyuni, Endah (2018): Design modification of the Cilafap hotel fave building using the flat slab method, Vol.7, No.2 (2018), Journal of Engineering ITS

[4] https://www.google.com/search?q=Jalur+column+and+jalur+trop\&rlz=1C1CHBF idID862ID862\&source=1 nms\&tbm=isch\&sa=X\&ved=2ahUKEwjh5tCB6P3mAhXRXCsKHZeboQQQQQQQQ $\quad$ 12-01-2020 hours 17.05WIB

[5] Imran, Iswandi, Fajar Hendrik (2016): Advanced planning of reinforced concrete structures, ITB press

[6] Kai, Qian. Bing, li (2013): Experimental study of the drop panel effects on response of reinforced concrete flat slabs after loss of corner column (Experimental and analytical analysis), DR-NTU, Nanyang Tecnological University Library, Singapore

[7] Kharis, Hasan Al (2019): Final alternative design of top structure using reinforced concrete with an outringger system, Mercu Buana University, Jakarta

[8] Lande, Pradip.S. Aniket B.Raut (2015): Seismic Behavior of flat slab systems, Vol. 2, April 10-June 2015 pp. 7-10, Journal of civil engineering and environmental technology, India

[9] Primakov, Anthones. Leo, Edison (2019): Study of the efficiency of flat slab systems with post-tension \& conventional methods, Vol.2, No.1 February 2019: pp 133-142, Journal of civil engineering partners

[10] Pratomo, Aloysius Hario Widhi (2017): Study of the efficiency of using flat slabs with drop panels against conventional slabs in a 5-story building, Universitas 17 Agustus 1945 samarinda

[11] Riswanto, Fery (2019): Final project of alternative comparison of reinforced concrete structure design in thamrin nine mansion apartment building in central Jakarta, Mercu Buana University, Jakarta

[12] Rosdiana. R, Ririn (2017): Final project design modification of golden hotel essential tulip structures using the flat slab method, Institut Teknologi November 10, Surabaya

[13] Syamsi, Muhammad Ibnu (2015): Comparison of two way slab with beam and flat slab analysis (case study: Coalyard West Kalimantan PLTU), Vol. 18, November 2, 2015: pp. 168-175, Scientific universe scientific journal

[14] Syarif, Harriad Akbar. Zulfikar Djauhari (2019): Structural response of the flat slab-drop panel structure of the irregular building to the earthquake load by analyzing the response of the spector, Vol.11, No.2 July 2015, Bina Widya University, Riau

[15] Wijaya, Tohar. Tedianto, Leo.S (2019): Analysis of the influence of the use of drop panels on the value of the bending moment and shear of the flat slab punch by the finite element method, Vol.2, May 2, 2019: pp. 125 - 134, Journal of civil engineering partners, Jakarta

\section{Biographies}

Agyanata Tua Munthe was born in March 21, 1981. After graduation his highschool education at BPK Penabur senior high school in Bandar Lampung, continued his civil engineering education at Atmajaya University, Special Region of Yogyakarta in 2004. He obtained his Master's degree in civil engineering in 2006 at University of Gadjah Mada, Special Region of Yogyakarta. Currentlt active as lecturer at Mercu Buana University, Jakarta as a lecturer with a concentration in structure and currently active as a project manager handling several construction project.

Guntur Jatmiko was born in Special Region of Yogyakarta on January 31, 1996. After graduation a vocational high school education in building engineering department continued his undergraduate civil engineering education at Mercu Buana University Jakarta in 2016 to 2020 by compiling the final project is Planning parking building using flat slab and drop panel as a replacement conventional beam with analyzing bending moment value and sliding style based on SNI 1726-2012. Aalso active as a staff in building construction projects. 